fœtus. If all go on well, it is best to proceed gently to fully dilate the os, without using any undue amount of force, though, in the event of hæmorrhage, it may be necessary to deliver before the os is fully dilated ; and, when it is well dilated, if the pains be rapid and strong, the membranes, if not previously ruptured, may be torn, and the case left to nature, as in cases Nos. 3 and 7. Such cases, however, are exceptional; and, as a rule, it will be desirable to expedite matters, as the partial separation of the placenta has an asphyxiating effect on the fotus, and the sooner it is born the better. The means to be employed will depend on circumstances. If the head be within reach, the forceps may be used, as was the case in three of my cases; more generally, version will be found suitable, simple traction being used in one breech-case.

To sum up: In all cases of placent prævia after the seventh month, labour should be induced, and previously to that, if the flooding be frequent or severe; labour should be induced-by dilatation of the os, the placenta separated sufficiently to permit flaccidity, and the dilatation proceeded with to the full extent. If not contra-indicated, ergot should then be given, and the child removed by the most rapid and gentle method. Such are the remarks I have to present on the treatment of placenta previa; and, while they contain nothing particularly novel or original, and fifteen cases are too few to dogmatise on, still they are sufficient to fairly test a method which I have hitherto found successful, and I trust I have stated the line of treatment clearly and distinctly, so as to leave no room for hesitation for those who will follow it when called upon to act.

In all the cases, as soon as the placenta was separated sufficiently, the hæmorrhage ceased completely, or became so very trivial as to be of no consequence.

P.S.-Since the Liverpool meeting in August, two more cases have come under treatment (making a total of seventeen); in each, labour was induced at the eighth month; both mothers recovered rapidly, and both children were born alive, one of which survives. For these cases, I am indebted to Drs. Fell and Beattie.

\section{ON THE UNITY OF POISON IN DISEASES.}

Read in the Section of Public Medicine.

BY G. DE GORREQUER GRIFFITH, L.R.C.P., Founder of, and late Benior Physician to, the Hospital for Women and Children.

In the brief space necessarily allotted each member, it would not be possible for me to do more than give a very short summary of this subject.

In the year 1875, I first called attention to the fact, that what is termed scarlatina occurring in the puerperal is frequently not scarlatina at all. But, before I proceed further, I must explain what I mean. I hold that there are two forms of scarlatina: the orthodox - viz., that contracted from a previously affected person, or something infected thereby-and the septicæmic, or toxæmic-viz., that which is evoluted or generated de novo, as a result of autogenetic blood-poisoning, such astoccurs in puerperal cases, or is evolved as a consequence of hetero-genetic blood-poisoning, such as arises (where no scarlatina had existed before) from drains, sewers, imbibition or ingestion of deleterious articles of drink-water, milk, cream, etc.or of food; and from decomposing animal or vegetable substances. This toxæmic or septicæmic scarlatina, which is evoluted from the above causes, is identical with the "surgical scarlatina" of Paget; and will on strict investigation, be found to be the form of scarlatina which more usually prevails among puerperals. In saying, "It is not scarlatina at all,"I mean it is not the orthodox-namely, that which is recognised by all the profession; and is, as all agree, contracted from a previously infected person or thing.

While carrying on this investigation, I was struck with the unity of origin in cases of puerperal fever, and typhoid also; and, in pursuing the inquiry, found how, from the same source, there would spring erysipelas; scarlatina, typhoid, puerperal, and diphtheria fevers; sore umbilicus in infants, sore eyes, sore mouth, and sore throats, embracing a very wide area of inflammation and inflammatory conditions of palate, tonsils, pharynx; and, passing stil further on in the respiratory tract, the larynx, the bronchi, the pleuræ, or the pleuræ and lung, would be found involved so that toxæmic or septicæmic laryngitis, bronchitis, pleurisy, pneumonia, and pleuropneumonia, attacking a variety of persons, may all, as in the case of the other forenamed ailments, viz., toxæmic, typhoid, etc. - as I call them to distingish them from the orthodox- be traced to the same causes; as could also hepatitis, diarrhœa, dysentery, cholera, enteritis, hæmorrhoids, and a large number of affections hitherto considered to be utterly distinct, and having no connection with each other, no point in common, no place where all meet together, because they diverge so much afterwards. Hence I formulated, as a heading to my papers, unity (as regards origin) of poison in the diseases I have just enumerated, and in many others, usually considered to be separate and entirely distinct.

This unity and differentiation in the course of evolution is now recognised by many medical men. Moreover, I found one disease (I adhere to the current word), or, as I term it, one set of symptoms, to be evoluted from another, in some cases so far apart in nosological arrangement as to be said to be wholly and entirely distinct, distinct even to specificity; and a number of nosologically divider diseases (sets of symptoms) to be evoluted from the one unity-point, the one common ancestry-differentiated no matter how widely, as they actually become, afterwards. I have, therefore, extended the formulated heading into "Unity of poison in diseases usually considered to be separate and entirely distinct; and the evolution of one from another apparently quite distinct, and of many diseases-seemingly all different-from one unity or common origin ;" evolution from this unity leading to differentiations. Further, I recognise that symptoms occurring in the course of an affection, say such as scarlatina, symptoms which ordinarily are termed "complications," may become primary motors of disease, originating a fresh epidemic, it may befresh, inasmuch as it would differ from that in the course of which it was itself evoluted. For example: Diphtheria not unfrequently is met with as a " complication" of scarlatina (I would say it was part and parcel of the scarlatina), which, in this instance, would be spoken of as the primary disease, the other as being secondary; this latter would be conveyed to a number of persons, even to such a number, it may be, as to constitute an epidemic, spreading fast from one to another. Then this diphth $r$ a would cease to propagate itself, or would cause something quite different to be evoluted, or would cause diphtheria to be evoluted in the persons exposed, or in the course of these evolutions it would hark back to that which set it agoing-viz., scarlatina; or there would originate or evolute, in a number of the exposed, certain other symptoms, usually considered sequelæ, but not actually part of the scarlatina; so that another outburst of something, seemingly altogether different from scarlatina and diphtheria, would take place, to canse perhaps another new evolution; or, again, to hark back to scarlatina or diphtheria, or both combined, as it would be termed; or would, as it were, leap forward and cause to be evoluted, in a certain number of cases, a very ulterior sequelæ of scarlatina-say rheumatism, or rheumatic fever, and heart and pericardic ${ }^{1}$ affections; or the latter per $s e$; or, it may be, nephritis (the scarlatinal form) would be evoluted, without any other intermediate symptoms being noticed, or any preexisting warning having been detected, or indeed been noticeable or detectable, such a form of nephritis as would be infectious-infective nephritis, I would call it-inasmuch as it has the power to set up scarlatina, or diphtheria, or any other ailment or group of symptoms coming within the range of its infectivity. In this way, new sets of symptoms may be evoluted, new outward manifestations of the inward workings or evolution, a new disease in fact, as would be said in current medical phraseology. In this I am fortified by the Bradshawe Lecture of Sir James Paget, and his and Sir William Gull's addresses on "Collective Investigation of Disease."

By unity of poison, I would beg my hearers to bear in mind I mean, not that the poison is always the same, but that the one poison-the one origo mali-whatever it may be, will originate several so-called different affections-widely different seemingly, widely different they would be spoken of, widely different even to be considered in every respect "specific."

1 See Dr. Sansom's recent Lettsomian Lectures, in which is confirmation of this.

A LITTLE KNOWLEDGK.-A candidate at a recent examination for a scholarship under the School Board is reported to have made the following statement concerning the process of digestion: "The food passes through the liver, and evaporates by means of little holes called capillaries." The examiner who published the answers suggests that this novel theory had its origin in the study of some cheap diagram of the digestive system. Knowledge of this kind is indeed a dangerous thing, and worse than conscious ignorance. There is a simple ignorance, and there is a compound ignorance, that is, there are people who are ignorant and know that they are; and there are people who are ignorant and think they know. The latter only are a dangerous class. 